\title{
Electrochemical oxidation of sulfamethazine on a glassy carbon electrode modified with graphene and gold nanoparticles
}

\author{
Ivana Cesarino $^{\mathrm{a}, *}$, Rafael Plana Simões ${ }^{\mathrm{a}}$, Francisco Carlos Lavarda ${ }^{\mathrm{b}}$, Augusto Batagin-Neto $^{c}$ \\ a College of Agricultural Sciences, UNESP-Univ Estadual Paulista, Campus Botucatu, Department of Bioprocess and Biotechnology, 18603-970 Botucatu, SP, \\ Brazil \\ ${ }^{\mathbf{b}}$ Faculty of Science, UNESP-Univ Estadual Paulista, Campus Bauru, Department of Physics, 17033-360 Bauru, SP, Brazil \\ c Campus Experimental de Itapeva, UNESP-Univ Estadual Paulista, 18409-010 Itapeva, SP, Brazil
}

\section{A R T I C L E I N F O}

Article history:

Received 29 October 2015

Received in revised form 5 January 2016

Accepted 22 January 2016

Available online 24 January 2016

\section{Keywords:}

Sulfamethazine

Oxidation mechanisms

Electronic structure calculations

Fukui indexes

Density Functional Theory

\begin{abstract}
A B S T R A C T
This work presents a comprehensive investigation of the oxidation mechanism of sulfamethazine (SMZ) combining electrochemical experiments and molecular modelling techniques. Cyclic voltammetry and differential pulse voltammetry experiments were performed in phosphate buffer solution (PBS) using a glassy carbon (GC) electrode modified with reduced graphene oxide and gold nanoparticles (rGO-AuNPs). Molecular modelling studies were performed via Density Functional Theory (DFT) employing Becke's LYP (B3LYP) exchange-correlation functional and the $6-31 \mathrm{G}(\mathrm{p}, \mathrm{d})$ basis set. The evaluation of molecular reactivity was accomplished by Condensed-to-Atoms Fukui Indexes (CAFIs). In the theoretical studies, three species were analysed: natural SMZ $\left(\mathrm{SMZ}^{(0)}\right)$ and its protolytic structures, $\mathrm{SMZ}^{(+\mathrm{H})}$ and SMZ ${ }^{(-\mathrm{H})}$. The $\mathrm{CV}$ results show a well-defined irreversible SMZ oxidation peak at $+0.89 \mathrm{~V}$. The molecular modelling studies indicate that $\mathrm{SMZ}^{(0)}$ is the species that effectively participates in the oxidation process. Based on the reactivity indexes obtained, two distinct oxidation mechanisms associated with EC processes occurring in the systems were proposed.
\end{abstract}

(c) 2016 Elsevier Ltd. All rights reserved.

\section{INTRODUCTION}

The focus of the research on environmental water quality is becoming more extensive, so, in addition to the classic pollutants, pharmaceutical products have been studied. Approximately 3000 different substances are applied in pharmaceutical formulations and their use has increased in recent decades [1]. In particular, antibiotics can cause an ecotoxicological problem, because of their potential persistence in the environment. Due to these environmental concerns, effluent treatment methodologies and adequate analytical strategies, with the goal of determining these antibiotics at low levels of concentration, are extremely important. One of these antibiotics is the sulfamethazine (SMZ), a substance widely used in pig farming effluent. The SMZ behavior and its modifications in electrocatalytic processes have been extensively investigated [2-4].

In this way, graphene has attracted much scientific and technological interest since its discovery in 2004. In particular, the extraordinary electronic transport properties, high electrocatalytic activities and large surface area of graphene make it a

\footnotetext{
* Corresponding author.

E-mail address: ivana@fca.unesp.br (I. Cesarino).
}

promising material for electrochemical applications [5]. Recently, several reviews with an emphasis on graphene-based electrochemical applications have been published [6-9]. Similarly to other materials based on carbon, graphene can also be employed as a support that can be modified with different species. In particular, gold nanoparticles (AuNPs) have unique properties such as good biological compatibility and conductivity and they can enhance the sensitivity and stability of graphene-modified electrodes. Thus, AuNPs have been incorporated into reduced graphene oxide (rGO) for the determination of SMZ.

One of the main challenges for electrochemical analysis is describing the mechanism associated with the oxidation/reduction processes that take place in the system. In most cases such a description is not possible by employing only electrochemical techniques. In this context, molecular modelling has proven to be a valuable tool. In general, such techniques enable identification and evaluation of the structural, electronic and reactivity features of molecular systems, and often allow the mechanisms involved to be confirmed [10-12]. As a matter of fact, it has been shown that molecular modelling methods and cyclic voltammetry techniques can be associated with understanding the redox mechanism of electroactive species [13-17]. The results of this kind of study may also be extended to drugs design $[18,19]$. 
In this report, voltammetric studies were performed on SMZbased systems in conjunction with electronic structure calculations. As exposed before, this important interaction between theoretical and experimental studies can provide more information to propose and to conclude the possible oxidation pathways. In this way, cyclic voltammetry and differential pulse voltammetry techniques were employed using a GC electrode modified with rGO-AuNPs composite. Frontier energy levels and reactivity indexes of a distinct SMZ-based system were evaluated to determine chemical species and molecular sites that effectively participate in charge transfer processes as well as possible chemical reactions that take place during SMZ oxidation. The results suggest that $S M Z$ suffers an irreversible oxidation at $+0.89 \mathrm{~V}$ that can be associated with two distinct electrochemical (EC) processes: i) formation of hydroxylamine-based derivatives $\left(\mathrm{SMZ}^{*}\right)$, and ii) possible formation of hydrazo-dimers (HD-SMZ).

\section{EXPERIMENTAL}

\subsection{Apparatus and procedures}

Cyclic voltammetry (CV) and differential pulse voltammetry (DPV) experiments were performed using a model PGSTAT30 Autolab electrochemical system (Eco Chemie, Utrecht, Netherlands) equipped with GPES software (Eco Chemie, Utrecht, Netherlands). The cell was assembled with a conventional threeelectrode electrochemical system: GC (glassy carbon)/rGO-AuNPs as a working electrode (diameter: $3 \mathrm{~mm}) ; \mathrm{Ag} / \mathrm{AgCl} / \mathrm{KCl}(3.0 \mathrm{~mol}$ $\mathrm{L}^{-1}$ ) as a reference electrode; and a Pt plate as an auxiliary electrode. All experiments were carried out at $25^{\circ} \mathrm{C}$. Electrochemical characterization of the $\mathrm{GC} / \mathrm{rGO}$-AuNPs electrode was performed using $\mathrm{CV}$ in $0.1 \mathrm{~mol} \mathrm{~L}^{-1} \mathrm{H}_{2} \mathrm{SO}_{4}$ with a scan rate of $50 \mathrm{mV} \mathrm{s}^{-1}$. DPV measurements were obtained with a scan rate of $10 \mathrm{mV} \mathrm{s}^{-1}$, pulse amplitude of $75 \mathrm{mV}$, and a step potential of $2 \mathrm{mV}$ in a $0.1 \mathrm{~mol} \mathrm{~L}^{-1}$ phosphate buffer solution (PBS) $\mathrm{pH} 8.0$, containing $5.0 \mu \mathrm{mol} \mathrm{L}^{-1}$ of SMZ standards.

The surface morphology of rGO-AuNPs composite was characterized using a field emission gun scanning electron microscope (FEG-SEM) and the images were recorded using a model FEI Inspect F50 microscope (FEI Company, Hillsboro, USA).

\subsection{Chemicals and solutions}

All solutions were prepared with water purified from a Barnstead Nanopure system, with resistivity $\geq 18 \mathrm{M} \Omega \mathrm{cm}$ (Thermo Scientific, USA). All reagents used in this study were of analytical grade and were used without further purification. Graphite powder of $1-2 \mu \mathrm{m}$ particle size, chloroauric acid $\left(\mathrm{HAuCl}_{4}\right)$ and SMZ were purchased from Sigma-Aldrich (Germany).

\subsection{Synthesis of the GO and rGO-AuNPs composite}

Graphene oxide (GO) was prepared by an improved Hummers' method [20]. Typically, $10 \mathrm{~g}$ of graphite and $10 \mathrm{~g}$ of $\mathrm{NaNO}_{3}$ was mixed with $450 \mathrm{~mL}$ of $\mathrm{H}_{2} \mathrm{SO}_{4}(98 \%)$ in a $2500 \mathrm{~mL}$ flask. The mixture was then stirred for $30 \mathrm{~min}$ in an ice bath, followed by the addition of $60 \mathrm{~g}$ of $\mathrm{KMnO}_{4}$ under vigorous stirring. The reaction mixture was then held under refrigeration at $4^{\circ} \mathrm{C}$ for $24 \mathrm{~h}$. Next, the mixture was stirred at $35^{\circ} \mathrm{C}$ followed by the addition of $450 \mathrm{~mL}$ of $\mathrm{H}_{2} \mathrm{O}$, also under vigorous stirring. After that, the reaction temperature was rapidly increased to $98^{\circ} \mathrm{C}$ and a further $450 \mathrm{~mL}$ of $\mathrm{H}_{2} \mathrm{O}$ was added, followed by $300 \mathrm{~mL}$ of $30 \% \mathrm{H}_{2} \mathrm{O}_{2}$ aqueous solution. The reaction mixture was again held under refrigeration at $4{ }^{\circ} \mathrm{C}$ for $24 \mathrm{~h}$. The obtained GO solution was centrifuged for $30 \mathrm{~min}$ at $8000 \mathrm{rpm}$ and washed several times with $5 \% \mathrm{HCl}$ and distilled water until the $\mathrm{pH}$ of the filtrate was neutral. Finally, the GO obtained was lyophilized for $24 \mathrm{~h}$.

The synthesis of the rGO-AuNPs composite was performed by microwave-assisted hydrothermal (MAH) method [21]. A suspension containing about $80 \mathrm{~g}$ of GO was mixed with $60 \mathrm{~mL}$ of ethylene glycol and sonicated for $15 \mathrm{~min}$. Then, $1 \mathrm{~mL}$ of $0.1 \mathrm{~mol} \mathrm{~L}^{-1}$ of a chloroauric acid solution was added to the suspension and sonicated for a further $10 \mathrm{~min}$. After that, the $\mathrm{pH}$ of the suspension was adjusted to 9 by adding $\mathrm{NaOH}$. The suspension was transferred to the reactor and the synthesis occurred at $140^{\circ} \mathrm{C}$ for $16 \mathrm{~min}$. The rGO-AuNPs obtained were centrifuged for $10 \mathrm{~min}$ at $3000 \mathrm{rpm}$ and washed several times with distilled water until the solution became colourless. After that, $5 \mathrm{~mL}$ of ethanol (pure grade) was added to the resulting composite and then the rGO-AuNPs were dried at $60^{\circ} \mathrm{C}$.

\subsection{Preparation of the GC modified with rGO-AuNPs composite}

Prior to modification, the GC electrode surface was polished with $0.3 \mu \mathrm{m}$ alumina slurries, rinsed thoroughly with doubledistilled water, sonicated for $5 \mathrm{~min}$ in ethanol and $5 \mathrm{~min}$ in water, and then dried in air. A suspension containing $1.0 \mathrm{mg}$ of rGO-AuNPs and $1.0 \mathrm{~mL}$ of water was dispersed using ultrasonic stirring for $20 \mathrm{~min}$. A $10 \mu \mathrm{L}$ aliquot of this dispersion was dropped onto the GC electrode surface, and dried at room temperature.

\subsection{Theoretical methods}

Fig. 1 shows the structure of natural $\mathrm{SMZ}\left(\mathrm{SMZ}^{(0)}\right)$ and its protolytic species $\left(\mathrm{SMZ}^{(+\mathrm{H})}\right.$ and $\left.\mathrm{SMZ}^{(-\mathrm{H})}\right)$. In general, there is a twostep dissociation pathway of sulfonamides involving the incorporation and release of protons [3,22].

For geometry optimization, 200 conformers of $\mathrm{SMZ}^{(0)}$ structure (Fig. 1) were constructed by two distinct approaches: randomly distributed dihedral angles (RDDA) and molecular dynamics (MD) calculations at elevated temperature with the aid of a Gabedit computational package [23] (see ref [24] for details). RDDA structures were obtained via a specific proprietary program.

All the conformers were then pre-optimized via the PM6 semiempirical method in a Hartree-Fock (HF) approach with the aid of the MOPAC2012 computational package [25]. The presence of the solvent was simulated via the Conductor-like Screening ModelCOSMO (with $\varepsilon=80.1$ ). After pre-optimizations, the most stable conformer was fully optimized via Density Functional Theory (DFT) calculations. Becke's LYP (B3LYP) exchange-correlation functional and $6-31 \mathrm{G}(\mathrm{p}, \mathrm{d})$ basis set were employed. The Polarizable Continuum Model (PCM) was employed for simulating the presence of the solvent at this stage. From the final optimized
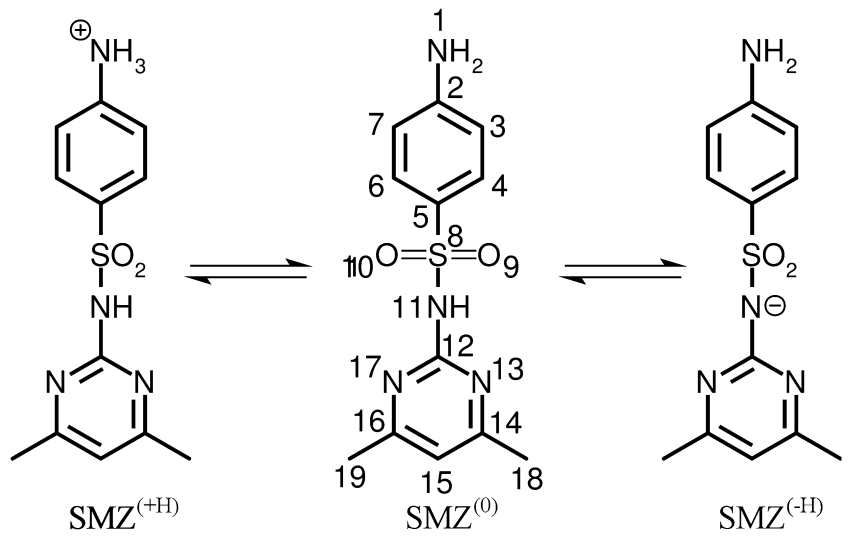

Fig. 1. Protolytic species of SMZ. 
geometry of $\mathrm{SMZ}^{\circ}$ the modified structures $\mathrm{SMZ}^{(+\mathrm{H})}$ and $\mathrm{SMZ}^{(-\mathrm{H})}$ were constructed and (directly) optimized at the DFT/B3LYP/PCM/ 6-31G(p,d) level.

The evaluation of molecular reactivity was accomplished by Condensed-to-Atoms Fukui Indexes (CAFIs) [26]. In general, such parameters carry information regarding local tendencies to insert electrons into or withdraw them from molecules, enabling identification of the sites that are most susceptible to undergoing electrophilic, nucleophilic or radical reactions [27]. Depending on the nature of the reaction, three distinct CAFIs can be defined [28]:

$\mathrm{f}_{\mathrm{k}}^{+}=\mathrm{q}_{\mathrm{k}}(\mathrm{N}+1)-\mathrm{q}_{\mathrm{k}}(\mathrm{N})$ for nucleophilic attack on atom $k$

$\mathrm{f}_{\mathrm{k}}{ }^{-}=\mathrm{q}_{\mathrm{k}}(\mathrm{N})-\mathrm{q}_{\mathrm{k}}(\mathrm{N}-1)$ for electrophilic attack on atom $k$

$\mathrm{f}_{\mathrm{k}}{ }^{\circ}=1 / 2\left[\mathrm{q}_{\mathrm{k}}(\mathrm{N}+1)-\mathrm{q}_{\mathrm{k}}(\mathrm{N}-1)\right]$ for radical attack on atom $k$

where $q_{k}(N+1), q_{k}(N)$ and $q_{k}(N-1)$ represent, respectively, the electronic population on the $k$-th atom of anionic, cationic and neutral species of the studied compound (all of them calculated at the optimized geometry of the neutral species, which is relevant in the study). In the work described here, the CAFIs were obtained in a DFT/B3LYP/PCM/6-31G(p,d). Hirshfeld's population analysis was employed to avoid non-realistic negative CAFIs [26].

The energies of frontier orbitals: i) the highest occupied molecular orbital (HOMO) and ii) the lowest unoccupied molecular orbital (LUMO) were estimated, respectively, by the ionization potential (IP) and electron affinity (EA) of the species [29].

All the DFT calculations were performed with the aid of the Gaussian 09 computational package [30].

\section{RESULTS AND DISCUSSION}

3.1. Morphological and electrochemical characterization of the rGOAuNPs composite

The surface morphology of the GO and rGO-AuNPs composite samples was characterized by FEG-SEM, as shown in Fig. 2. Fig. 2(a) displays typical images of GO where it is evident that the material consists of a mixture of single layers and multi-layer graphene sheets. On the other hand, Fig. 2(b) presents the images of rGOAuNPs. The rGO sheets are wrinkled, presenting AuNPs with sizes varying between 5 and $30 \mathrm{~nm}$, showing that the AuNPs were attached to the rGO support.

The presence of Au nanoparticles on the rGO was also characterized electrochemically. As a result, CV experiments were carried out in $0.1 \mathrm{~mol} \mathrm{~L}^{-1}$ of $\mathrm{H}_{2} \mathrm{SO}_{4}$, with a scan rate of $50 \mathrm{mV} \mathrm{s}^{-1}$ in the potential range of 0.0 to $+1.6 \mathrm{~V}$. The electrochemical behaviour of the rGO-AuNPs composite was compared with the voltammetric response of a GC electrode modified with GO. In the cyclic voltammograms shown in Fig. 3, no electrochemical process was observed for the voltammetric response of the GC/GO electrode. However, the GC electrode modified with the rGO-AuNPs composite presented peaks at +1.14 and $+0.87 \mathrm{~V}$, which correspond to the Au oxidation and Au oxide reduction, respectively. Therefore, the voltammetric characterization confirmed the presence of $\mathrm{Au}$ nanoparticles in rGO.

\subsection{Electrochemical behaviour of GC/rGO-AuNPs composite electrode on the SMZ oxidation process}

The electrochemical behaviour of SMZ on the GG/rGO-AuNPs composite electrode was carried out in $0.1 \mathrm{~mol} \mathrm{~L}^{-1} \mathrm{PBS} \mathrm{pH} 8.0$,
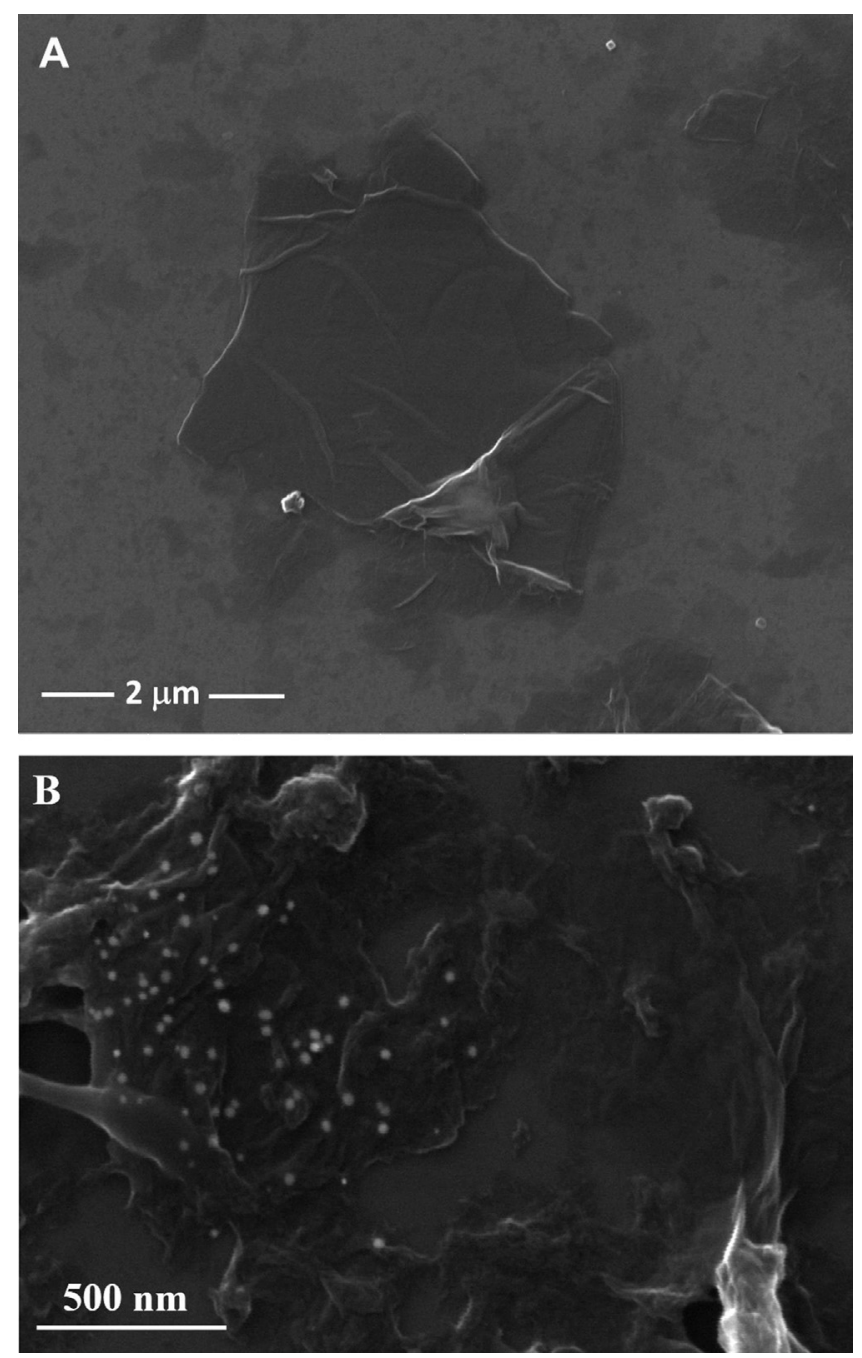

Fig. 2. FEG-SEM micrographs for: (a) GO and (b) rGO-AuNPs composite.

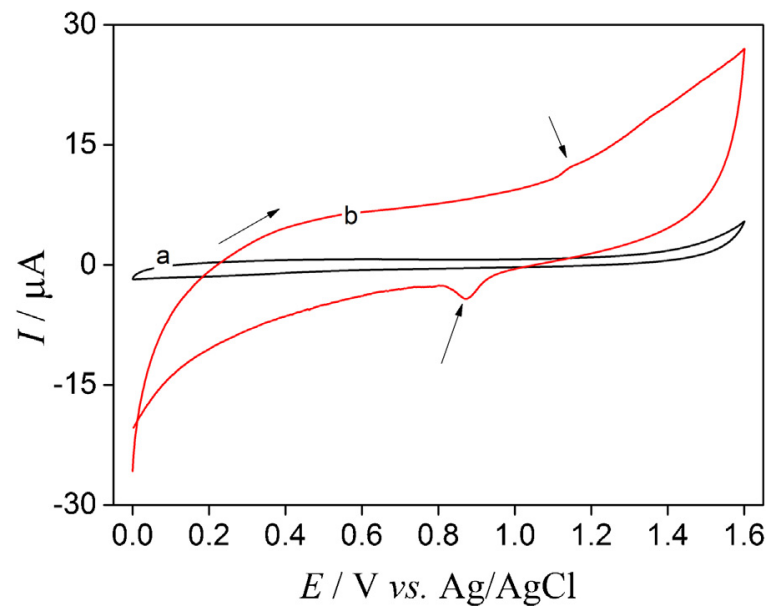

Fig. 3. Cyclic voltammetry characterization in $0.1 \mathrm{~mol} \mathrm{~L}^{-1}$ of $\mathrm{H}_{2} \mathrm{SO}_{4}$ with a scan rate of $50 \mathrm{mV} \mathrm{s}^{-1}$ for the electrodes: (a) GC/GO and (b) GC/rGO-AuNPs.

containing $50.0 \mu \mathrm{mol} \mathrm{L}^{-1} \mathrm{SMZ}$, by cyclic voltammetry experiments, with a scan rate of $50 \mathrm{~m} \mathrm{~V} \mathrm{~s}^{-1}$. The results are presented in Fig. 4. The two electrochemical processes observed in the voltammetric response in the absence of SMZ (dotted line) are due to the Au oxidation and Au oxide reduction. When the SMZ is 


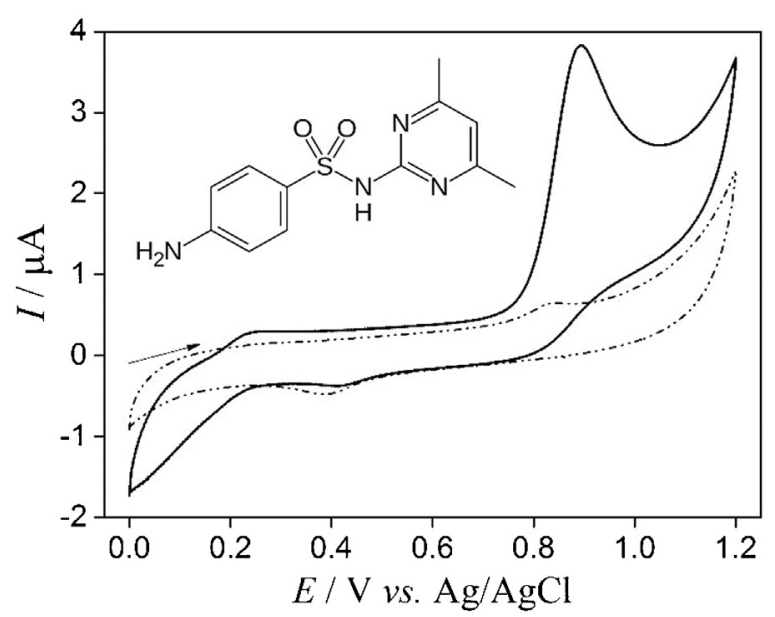

Fig. 4. CV scans of the $\mathrm{GC} / \mathrm{rGO}$-AuNPs composite electrode in $0.1 \mathrm{~mol} \mathrm{~L}^{-1} \mathrm{PBS} \mathrm{pH}$ 8.0 in the absence (dotted line) and in the presence (solid line) of $50.0 \mu \mathrm{mol} \mathrm{L}^{-1}$ of SMZ with a scan rate of $50 \mathrm{mV}$ s-1. Inset: SMZ structure.

added into the solution (solid line), a well-defined irreversible oxidation peak at $+0.89 \mathrm{~V} v \mathrm{~s}$. $\mathrm{Ag} / \mathrm{AgCl} / \mathrm{KCl}\left(3.0 \mathrm{~mol} \mathrm{~L}^{-1}\right)$ is observed. The relative intensity and the peak position are not changed after consecutive CV cycles (not shown for simplicity). However, it is not possible to affirm which active site of the SMZ molecule is being oxidized and the mechanism involved in the process.

At first, the irreversibility of the process is not compatible with the maintenance of the CV spectra features after successive cycles. However, such persistent oxidation peak for consecutive CV cycles should be associated with the products generated by the SMZ oxidation mechanism in the first cycle, and the maintenance of the peak at $+0.89 \mathrm{~V}$ for consecutives cycles indicates that the products generated by the first $\mathrm{CV}$ cycle have the same oxidation potential as the molecules present in the initial condition of the experiment.

In order to provide the mechanism of SMZ oxidation on the $\mathrm{GC} / \mathrm{rGO}$-AuNPs composite electrode, the dependence of the electrochemical oxidation of SMZ on $\mathrm{pH}$ was studied by DPV experiments at pHs ranging from 5.0 to 10.0 in PBS containing $5 \mu \mathrm{mol} \mathrm{L}^{-1} \mathrm{SMZ}$. The results are shown in Fig. 5, which is a plot of the DPV peak current $\left(I_{\mathrm{pa}}\right)$ and peak potential $\left(E_{\mathrm{pa}}\right)$ as a function of $\mathrm{pH}$. The variation of $E_{\mathrm{pa}}$ with $\mathrm{pH}$ can provide valuable information about the SMZ oxidation process. Reducing the hydrogen ion concentration of the electrolyte causes a shift in peak potential for SMZ oxidation towards more negative values, as illustrated in Fig. 5. This is a consequence of deprotonation during the oxidation process, which is facilitated at higher $\mathrm{pH}$. The $E_{\mathrm{pa}} v s$. $\mathrm{pH}$ showed a

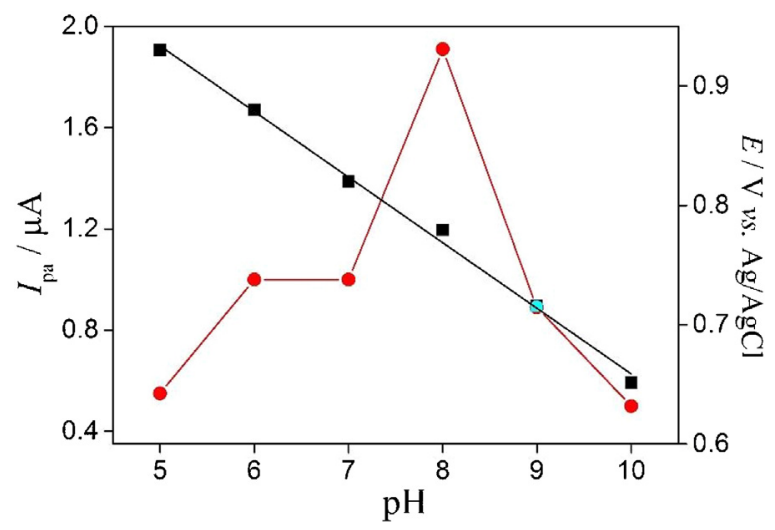

Fig. 5. Effect of pH on the peak potential $(\square)$ and peak current ( $(\bullet)$ for SMZ oxidation on the GC/rGO-AuNPs composite electrode using $0.1 \mathrm{molL}^{-1}$ PBS containing $5 \mu \mathrm{mol} \mathrm{L}^{-1}$ of SMZ. linear relationship, with a slope of $55 \mathrm{mV}$ per $\mathrm{pH}$ unit. Therefore, the electrochemical process involves the same number of protons and electrons during the electrooxidation of SMZ; however, it is still not possible to predict the SMZ oxidation mechanism. Thus, molecular modelling studies were performed. Even in Fig. 5, the plot of $I_{\mathrm{pa}} v s$. pH for SMZ shows that the major anodic peak current was obtained at $\mathrm{pH}$ 8.0.

Analysing the results from the CV and DPV experiments jointly, besides the well-defined oxidation peak at $+0.89 \mathrm{~V}$, a small oxidation peak at $+0.24 \mathrm{~V}$ in the CV scan can be noted. This peak can be understood from the DPV experiment and previous results from studies analysing the SMZ oxidation potential as a function of the solution $\mathrm{pH}$. Preliminary results reported in ref. [31] showed the dependence between the solution $\mathrm{pH}$ and the SMZ oxidation potential, in which the buffer solution $\mathrm{pH}$ ranged from 1.5-9.0. These results revealed that, by increasing the $\mathrm{pH}$, the oxidation peak potential of SMZ shifts to less positive potentials due to the hindrance of the oxidation at low proton concentrations. However, when the solution $\mathrm{pH}$ is neutral or close to neutral, two oxidation peaks can be observed probably due to the two acid-base centres of the SMZ, producing two new SMZ species in the solution. Everything indicates that different SMZ species may be present in the solution during the CV experiment: the SMZ in its natural state; the SMZ species resulting from its molecular interaction with the buffer solution ions; the product of the SMZ oxidized at low potentials $(+0.24 \mathrm{~V})$; and the product of the SMZ oxidized at the well-defined peak of $+0.89 \mathrm{~V}$.

\subsection{Molecular modelling studies}

Fig. 6 presents the most stable ground state structure and CAFI obtained for the species $\mathrm{SMZ}^{(0)}, \mathrm{SMZ}^{(+\mathrm{H})}$ and $\mathrm{SMZ}^{(-\mathrm{H})}$. Red and blue regions indicate very reactive and non-reactive sites, respectively. The other colours represent sites with intermediary reactivities, according to the following increasing order: blue, green, yellow, orange, red.

As can be seen, the formation of $\mathrm{SMZ}^{(+\mathrm{H})}$ and $\mathrm{SMZ}^{(-\mathrm{H})}$ species can be understood from $\mathrm{f}^{-}$and $\mathrm{f}^{+}$indexes of the SMZ precursor. The largest value of $\mathrm{f}^{-}$is observed in Nitrogen $\mathbf{1}$ (Fig. 1 ), suggesting that this atom is the most reactive site for reactions towards electrophilic agents ( such as $\mathrm{H}^{+}$), so, $\mathrm{SMZ}^{(+\mathrm{H})}$ species can be generated just by proton incorporation in the $\mathrm{N}_{1}$ atom of $\mathrm{SMZ}^{(0)}$. On the other hand, the results obtained for $\mathrm{f}^{+}$suggest that the formation of $\mathrm{SMZ}^{(-\mathrm{H})}$ species is not so direct, and probably involves additional chemical interactions of external species in atoms $\mathrm{N}_{13}$ and $\mathrm{N}_{17}$.

The CAFIs obtained for $\mathrm{SMZ}^{(-\mathrm{H})}$ species show ring $\mathrm{B}$ as the most reactive region towards nucleophilic agents and free-radical attacks, while $\mathrm{N}_{11}$ and $\mathrm{C}_{15}$ are the most reactive sites for reactions towards electrophiles. In particular, this last feature indicates that $\mathrm{SMZ}^{(-\mathrm{H})}$ species are promptly converted into $\mathrm{SMZ}^{(0)}$ in the presence of protons. With regard to $\mathrm{SMZ}^{(+\mathrm{H})}$ species, higher reactivity towards nucleophiles is observed on ring $A$, while $C_{15}$ and $\mathrm{N}_{11}$ are the most reactive sites for reactions towards nucleophiles, suggesting that additional species could be formed at very low $\mathrm{pH}$.

Among the three indexes presented in Fig. $6, \mathrm{f}^{-}$carries more relevant information regarding the oxidation of SMZ. This parameter describes the susceptibility of each atom to losing one electron, i.e. what the molecular position that is prone to undergoing oxidation processes.

In general, charge transfer (CT) processes depend on several factors, such as how effective the interaction is between donor molecules (SMZ derivatives in our case) and electrode surfaces, and how appropriate the energy level alignments are between them. 


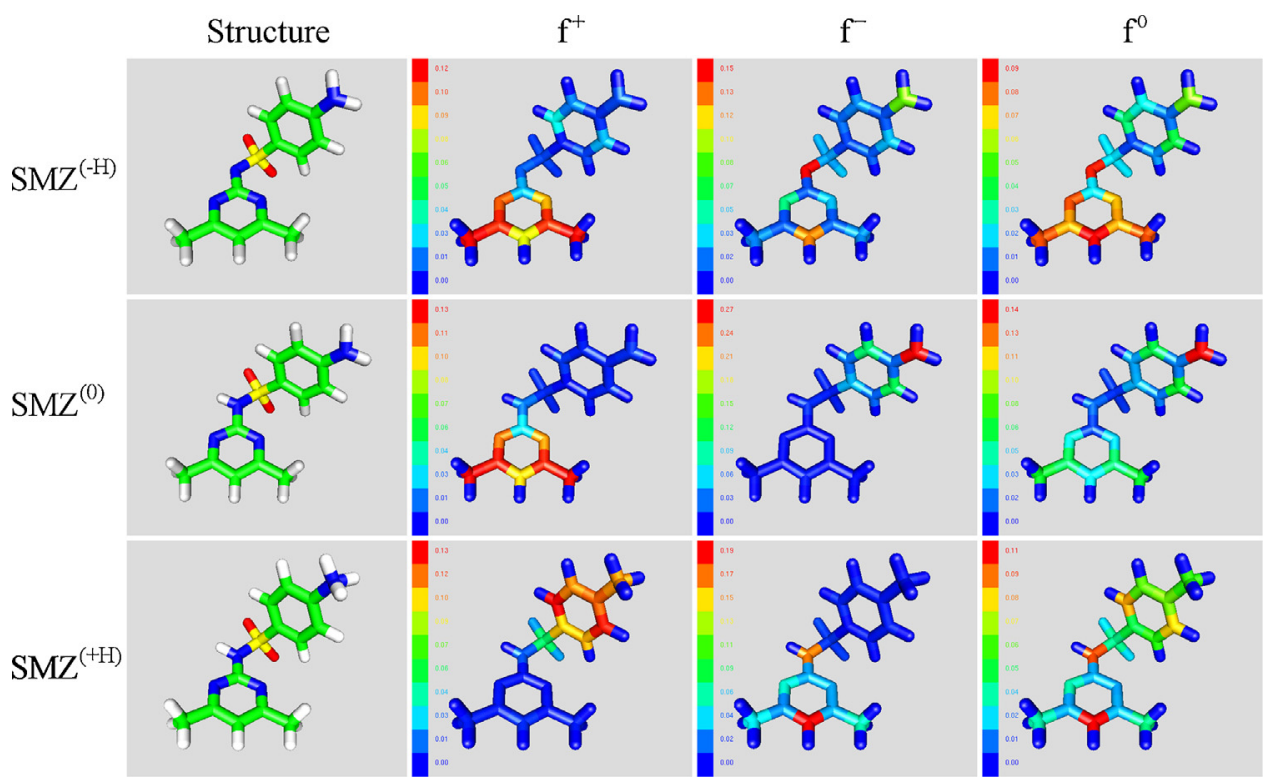

Fig. 6. Structures and CAFI values of protolytic species of SMZ.

With regard to molecule/electrode interactions, our results suggest that a more effective CT could be expected for the molecules $\mathrm{SMZ}^{(+\mathrm{H})}$ and $\mathrm{SMZ}^{(0)}$, since both of them present sites with higher $\mathrm{f}^{-}$values in terminal regions of their structures. After the adsorption of the molecules on the electrode's surface, it is expected that these (more accessible) regions would interact more efficiently with the electrodes than central (and hysterically protected) regions, resulting in more effective CT processes.

Fig. 7 presents the estimated energy levels of frontier orbitals of $\mathrm{SMZ}$ derivatives. The structures of $\mathrm{SMZ}^{(-\mathrm{H})}, \mathrm{SMZ}^{(0)}$ and $\mathrm{SMZ}^{(+\mathrm{H})}$ are presented in Fig. 1. SMZ*, HD-SMZ and AD-SMZ represent possible products coming from EC processes and will be discussed later in more detail. The grey region defines the energy range associated with the work function of graphene-based electrodes [32] and the dashed line illustrates an estimation of the work function of our composite electrode, $\mathrm{W}_{\mathrm{GC} / \mathrm{rGO} \text {-AuNPs }}$ (obtained from Fig. 4). The blue arrows represent the energy difference between $\mathrm{W}_{\mathrm{GC} / \mathrm{rGO} \text {-AuNPs }}$ and $\mathrm{E}_{\text {HOMO }}$ of the molecules $(\Delta \mathrm{E})$.

The results suggest that oxidation of SMZ-based systems can occur in three different regimes: $\mathrm{i}$ ) at very low voltages (below $0.5 \mathrm{~V}$ ) due to $\mathrm{SMZ}^{(-\mathrm{H})}$, ii) at intermediate voltages (around $0.75 \mathrm{~V}$ )

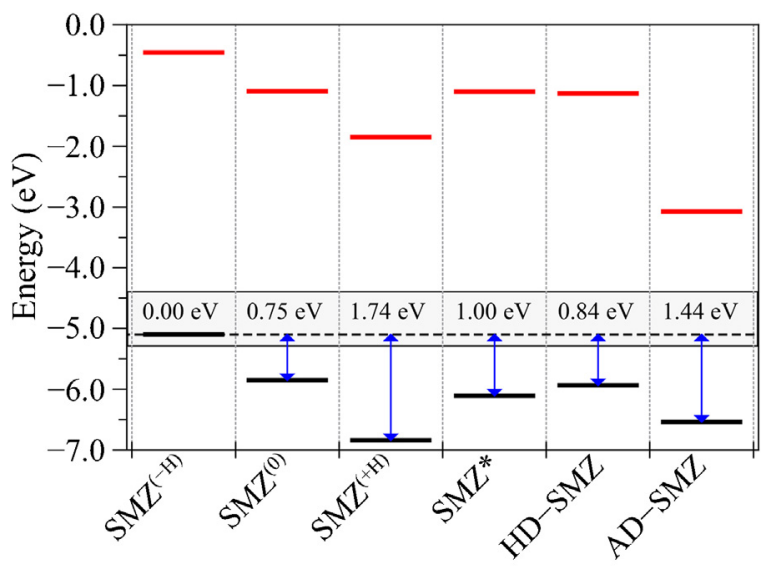

Structures

Fig. 7. HOMO and LUMO energy levels of SMZ derivatives. due to $\mathrm{SMZ}^{(0)}$, and iii) at higher voltages (above $1.75 \mathrm{~V}$ ) due to $\mathrm{SMZ}^{(+\mathrm{H})}$ species. Given the nature of the involved protolytic species, such signals should be expected at high, neutral and low $\mathrm{pH}$, respectively. In general, the energy differences observed are compatible with the dependence of voltage peak on the $\mathrm{pH}$, which suggests that lower voltages are necessary to promote oxidation at higher $\mathrm{pH}$.

With regard to $\mathrm{SMZ}^{(-\mathrm{H})}$, despite the observation of a small peak at $+0.24 \mathrm{~V}$, the absence of pronounced oxidation peaks at very low voltages indicates that the electron transfer from this species to the electrode is not effective, which could be associated with the central position of reactive sites in the structure of the molecule. On the other hand, the dependence of peak current on the $\mathrm{pH}$ indicates that the presence of $\mathrm{SMZ}^{(-\mathrm{H})}$ species does influence the oxidation, leading to higher currents. This effect, in conjunction with the absence of oxidation peaks at low voltages, suggests that although $\mathrm{SMZ}^{(-\mathrm{H})}$ species do not directly participate in the CT process, they could interact, for example, with $\mathrm{SMZ}^{(0)}$ species adsorbed on the electrode (which presented the most compatible $\Delta \mathrm{E}$ values), doping them and enhancing the peak current. In this context, at very high $\mathrm{pH}$, when a low concentration of $\mathrm{SMZ}^{(0)}$ species is expected, the efficiency of the process is reduced, due to non-effective interaction between $\mathrm{SMZ}^{(-\mathrm{H})}$ and the composite electrode, which promotes the quenching of the current peak observed in Fig. 5.

In the case of $\mathrm{SMZ}^{(+\mathrm{H})}, \Delta \mathrm{E}$ is higher than the maximum applied potential, so that this species could not effectively participate in the oxidation process. Additionally, the spectrum presented in Fig. 4 was taken at $\mathrm{pH}=8.0$, when a very low concentration of $\mathrm{SMZ}^{(+\mathrm{H})}$ is indeed expected, confirming that this species is not directly associated with the observed oxidation process.

Finally, among all the protolytic species, $\mathrm{SMZ}^{(0)}$ presents a $\Delta \mathrm{E}$ value that is more compatible with the experimental data, suggesting that this structure participates effectively in the oxidation process. Nevertheless, the absence of a reduction peak in the CV spectrum suggests that SMZ oxidation is probably accompanied by chemical reactions (EC processes), which turn it into an irreversible process. In order to better evaluate what kind of reaction could occur in the oxidized system, CAFIs were calculated for $\mathrm{SMZ}^{+}$and $\mathrm{SMZ}^{2+}$ (considering a simple of double oxidation of $\left.S M Z^{(0)}\right)$. Fig. 8 presents the results obtained. 


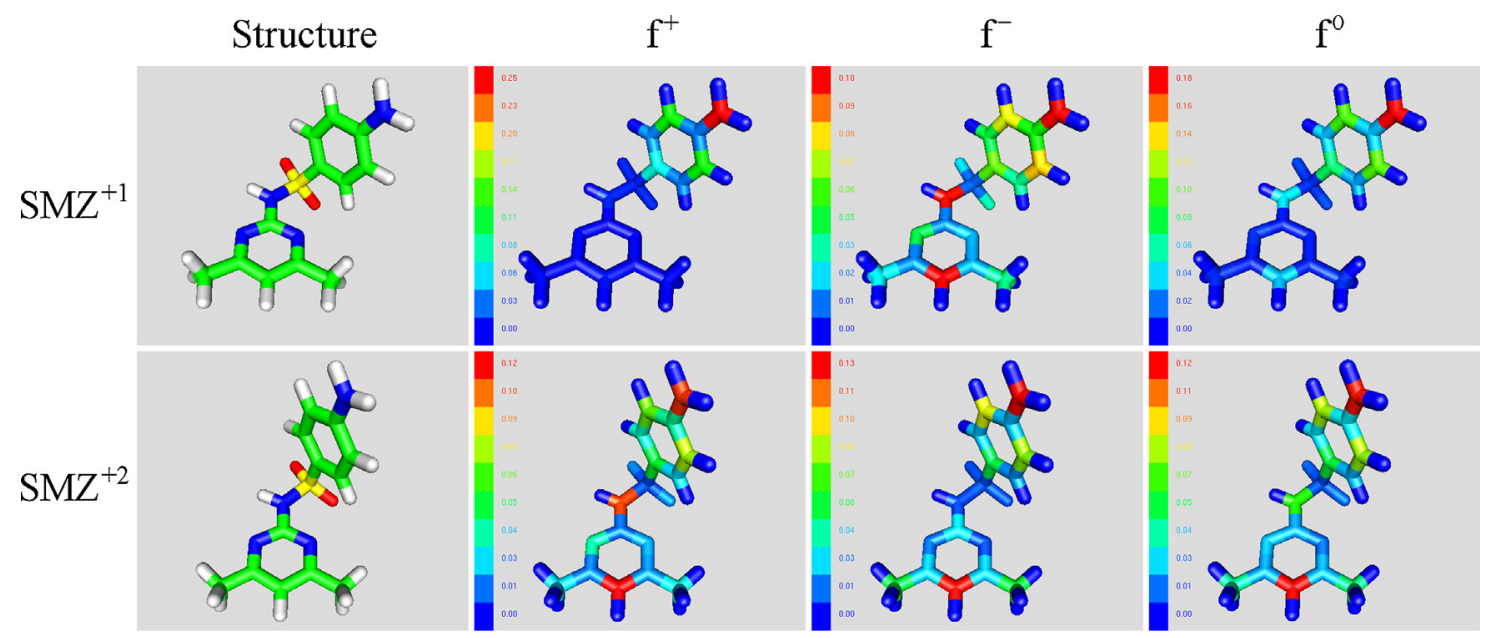

Fig. 8. Structures and CAFI values of oxidized species of SMZ.

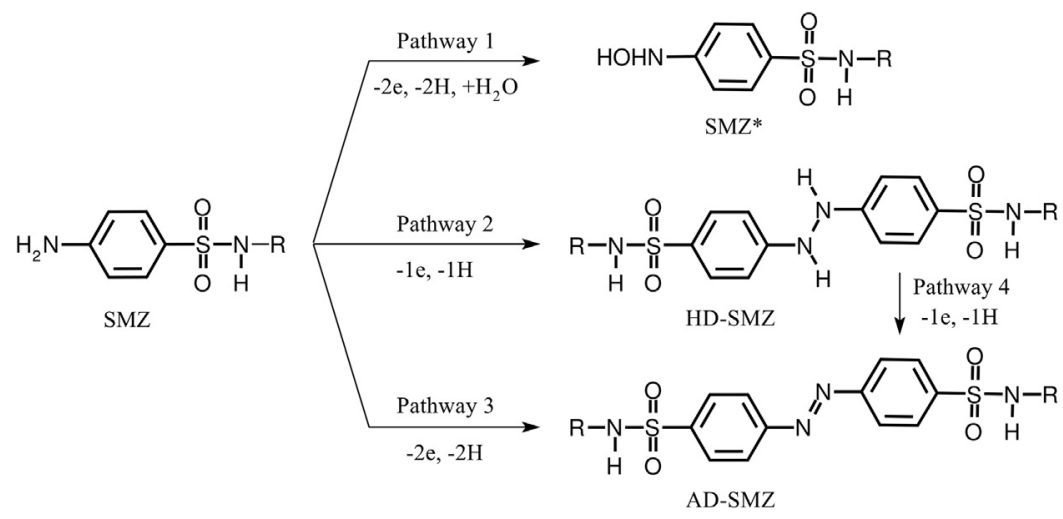

Fig. 9. Possible products coming from EC processes associated with the oxidation of SMZ.

Note that both $\mathrm{SMZ}^{+1}$ and $\mathrm{SMZ}^{+2}$ present high reactivity towards nucleophiles in $\mathrm{N}_{1}$, suggesting that $\mathrm{OH}^{-}$chemical agents could attack this region after SMZ oxidation, generating the SMZ* species (see Fig. 9, Pathway 1 and Fig. 7). As a matter of fact, the generation of similar species has already been proposed by Fotouhi and Zabeti for doubly oxidized SMZ-based systems [31]. In particular, the participation of two electrons and two protons in this EC process is compatible with was predicted by the Nernst Equation in our systems.

It is important to note that, since the $\mathrm{SMZ}^{*}$ species is generated in its neutral form, the reduction process cannot be observed in the system, which is compatible with our experimental results (Fig. 4). Additionally, given the $\Delta \mathrm{E}$ values associated with $\mathrm{SMZ}^{*}$ (around $1 \mathrm{eV}$ ), this structure is supposed to participate in the oxidation process in approximately the same potential range as $\mathrm{SMZ}^{(0)}$, which could be linked to the absence of significant quenching of the oxidation peak after repeated voltammetric cycles (also observed by Fotouhi and Zabeti [31]).

As a matter of fact, the generation of $\mathrm{SMZ}^{*}$ species has also been associated with the degradation of SMZ induced by gamma rays [33]. In their work, Liu and Wang suggest that $\mathrm{OH}$. radicals, coming from water radiolysis, attack the $\mathrm{SMZ}^{(0)}$ structure, leading to varied by-products, including SMZ*. Our results, however, suggest that a significant part of these by-products could also be associated with nucleophilic or electrophilic attacks on specific sites of SMZoxidized structures (Fig. 8), indicating a possible relevance of EC processes for $\mathrm{SMZ}$ radiolysis.
In addition to the mechanism associated with the production of hydroxylamine-based derivative SMZ*, the CAFI results also suggest the possibility of the formation of dimeric structures after $\mathrm{SMZ}^{(0)}$ oxidation. As can be seen in Fig. $8, \mathrm{~N}_{1}$ is the most reactive site for both nucleophilic and electrophilic attack on the structure of the oxidized species. In this context, bearing in mind the possibility of interactions between two oxidized SMZ species, it should be expected that dimeric structures could be formed from $\mathrm{N}-\mathrm{N}$ linkage (see Fig. 9, Pathways 2 and 3). Indeed, the formation of such hydrazo- and azo-dimers (HD-SMZ and AD-SMZ, whose energy levels are presented in Fig. 7) has already been proposed by Gao et al. in their study on SMZ oxidation in the presence of $\mathrm{MnO}_{2}$, at low pH [34]. Similarly to SMZ*, our results suggest that HD-SMZ structures can also participate in additional oxidation processes in the same potential range of the original system, which is not observed for AD-SMZ. However, in contrast to SMZ*, an efficient interaction could not be expected between more extended dimeric structures and the electrode, so more significant quenching should be observed after sequential scans. Additionally, a consumption of HD-SMZ species should also be expected from Pathway 4 presented in Fig. 9, again leading to CV signal quenching.

Despite of indicated by our CAFI results, it is important to highlight that the formation of such azo and hydrazo species demands some specific experimental conditions that commonly involves counterions interactions and proper pHs [34-37]. that could hinder the hypothesis of dimer formation in our system. However, given the number of variables involved, we consider that 
this hypothesis can not be completely ruled out, deserving more studies.

\section{CONCLUSION}

Cyclic voltammetry was employed to evaluate electrochemical processes of sulfamethazine on a glassy carbon electrode modified with graphene and gold nanoparticles. The results evidence an irreversible oxidation of SMZ at $+0.89 \mathrm{~V}$.

Reactivity indexes and frontier energy levels of distinct protolytic species of SMZ were evaluated via electronic structure calculations. The results suggest that the oxidation of SMZ is associated with the $\mathrm{SMZ}^{(0)}$ species. Based on the CAFI results, it was possible to propose the nature of the EC mechanisms probably associated with the irreversible oxidation of SMZ. The products of the oxidation can also oxidize in the same potential range of SMZ, which could explain the absence of significant quenching in the CV spectrum after successive scans.

\section{ACKNOWLEDGEMENTS}

We are grateful for financial support from FAPESP (grants 2015/ 02136-2) and CNPq. This research was also supported by resources supplied by the Center for Scientific Computing (NCC/GridUNESP) of São Paulo State University (UNESP).

\section{References}

[1] S.D. Richardson, Water analysis: emerging contaminants and current issues, Anal. Chem. 81 (2009) 4645-4677, doi:http://dx.doi.org/10.1021/ac9008012.

[2] M. Pérez-Moya, M. Graells, G. Castells, J. Amigó, E. Ortega, G. Buhigas, et al., Characterization of the degradation performance of the sulfamethazine antibiotic by photo-Fenton process, Water Res. 44 (2010) 2533-2540.

[3] A. El-Ghenymy, P.L. Cabot, F. Centellas, J.A. Garrido, R.M. Rodríguez, C. Arias, et al, Electrochemical incineration of the antimicrobial sulfamethazine at a boron-doped diamond anode, Electrochim. Acta 90 (2013) 254-264, doi: http://dx.doi.org/10.1016/j.electacta.2012.11.125.

[4] M.J. García-Galán, M.S. Díaz-Cruz, D. Barceló, Kinetic studies and characterization of photolytic products of sulfamethazine, sulfapyridine and their acetylated metabolites in water under simulated solar irradiation, Water Res. 46 (2012) 711-722, doi:http://dx.doi.org/10.1016/j.watres.2011.11.035.

[5] C. Gao, X.-J. Huang, Voltammetric determination of mercury(II), TrAC Trends Anal. Chem. 51 (2013) 1-12, doi:http://dx.doi.org/10.1016/j.trac.2013.05.010.

[6] D.A. Brownson, C.E. Banks, Graphene electrochemistry: an overview of potential applications, Analyst 135 (2010) 2768-2778.

[7] M. Pumera, A. Ambrosi, A. Bonanni, E.L.K. Chng, H.L. Poh, Graphene for electrochemical sensing and biosensing, TrAC Trends Anal. Chem. 29 (2010) 954-965.

[8] D. Chen, H. Feng, J. Li, Graphene oxide: preparation, functionalization, and electrochemical applications, Chem. Rev. 112 (2012) 6027-6053, doi:http://dx. doi.org/10.1021/cr300115 g.

[9] J.W. Yuyan Shao, Graphene Based Electrochemical Sensors and Biosensors: A Review, Electroanalysis 22 (2010) 1027-1036, doi:http://dx.doi.org/10.1002/ elan.200900571.

[10] B. Ramos, J.P.S. Farah, A.C.S.C. Teixeira, Estimating reaction constants by ab initio molecular modeling: a study on the oxidation of phenol to catechol and hydroquinone in advanced oxidation processes, Braz. J. Chem. Eng. 29 (2012) 113-120, doi:http://dx.doi.org/10.1590/S0104-66322012000100013.

[11] O. Borodin, Molecular Modeling of Electrolytes, in: T.R. Jow, K. Xu, O. Borodin, M. Ue (Eds.), Electrolytes Lithium Lithium-Ion Batter, Springer, New York, 2014, pp. 371-401. (accessed June 4, 2015) http://link.springer.com/chapter/ 10.1007/978-1-4939-0302-3_8.

[12] B. Ramos, A.C.S.C. Teixeira, Molecular-Scale Modeling of the Degradation of Phenol in Advanced Oxidation Processes Reaction Media, In: C.A.O. do N. and E. C.B. Rita Maria de Brito Alves (Eds.), Comput. Aided Chem. Eng., Elsevier, 2009: pp. 285-290. http://www.sciencedirect.com/science/article/pii/ S1570794609702688 (accessed June 4, 2015).

[13] J. Moens, P. Jaque, F. De Proft, P. Geerlings, The Study of Redox Reactions on the Basis of Conceptual DFT Principles: EEM and Vertical Quantities, J. Phys. Chem. A 112 (2008) 6023-6031, doi:http://dx.doi.org/10.1021/jp711652a.
[14] J. Faver, K.M. Merz, The Utility of the HSAB Principle via the Fukui Function in Biological Systems, J. Chem. Theory Comput. 6 (2010) 548-559, doi:http://dx. doi.org/10.1021/ct9005085.

[15] J. Melin, P.W. Ayers, J.V. Ortiz, Removing Electrons Can Increase the Electron Density: A Computational Study of Negative Fukui Functions, J. Phys. Chem. A 111 (2007) 10017-10019, doi:http://dx.doi.org/10.1021/jp075573d.

[16] E. de, S. Gil, C.H. Andrade, N.L. Barbosa, R.C. Braga, S.H.P. Serrano, Cyclic voltammetry and computational chemistry studies on the evaluation of the redox behavior of parabens and other analogues, J. Braz. Chem. Soc. 23 (2012) 565-572, doi:http://dx.doi.org/10.1590/S0103-50532012000300025.

[17] J. Guo, J. Lian, Y. Guo, X. Liu, C. Zhang, L. Yue, et al., Redox activity and accelerating capacity of model redox mediators during biodenitrification, Biotechnol. Biotechnol. Equip. 0 (2015) 1-7, doi:http://dx.doi.org/10.1080/ 13102818.2015.1027504.

[18] S. Rauf, J.J. Gooding, K. Akhtar, M.A. Ghauri, M. Rahman, M.A. Anwar, et al., Electrochemical approach of anticancer drugs-DNA interaction, J. Pharm. Biomed. Anal. 37 (2005) 205-217, doi:http://dx.doi.org/10.1016/j. jpba.2004.10.037.

[19] S. Prabhulkar, H. Tian, X. Wang, J.-J. Zhu, C.-Z. Li, Engineered Proteins: Redox Properties and Their Applications, Antioxid. Redox Signal. 17 (2012) 17961822, doi:http://dx.doi.org/10.1089/ars.2011.4001.

[20] S. Hummers William Jr., E. Offeman Richard, Preparation of Graphitic Oxide, J. Am. Chem. Soc. 80 (1958) 1339, doi:http://dx.doi.org/10.1021/ja01539a017.

[21] F.V. Motta, A.P.A. Marques, J.W.M. Espinosa, P.S. Pizani, E. Longo, J.A. Varela, Room temperature photoluminescence of BCT prepared by Complex Polymerization Method, Curr. Appl. Phys. 10 (2010) 16-20.

[22] N. Şanli, S. Şanli, G. Özkan, A. Denizlic, Determination of pKa values of some sulfonamides by LC and LC-PDA methods in acetonitrile-water binary mixtures, J. Braz. Chem. Soc. 21 (2010) 1952-1960, doi:http://dx.doi.org/ $10.1590 /$ S0103-50532010001000022.

[23] A.-R. Allouche, Gabedit-a graphical user interface for computational chemistry softwares, J. Comput. Chem. 32 (2011) 174-182, doi:http://dx.doi. org/10.1002/jcc. 21600 .

[24] A. Batagin-Neto, E.F. Oliveira, C.F.O. Graeff, F.C. Lavarda, Modelling polymers with side chains: MEH-PPV and P3HT, Mol. Simul. 39 (2013) 309-321, doi: http://dx.doi.org/10.1080/08927022.2012.724174.

[25] J.J.P. Stewart, MOPAC: A semiempirical molecular orbital program, J. Comput. Aided Mol. Des. 4 (1990) 1-103, doi:http://dx.doi.org/10.1007/BF00128336.

[26] A.R.S.J. Guadalupe Hernandez, Theoretical and experimental studies of phenol oxidation by ruthenium complex with $\mathrm{N}, \mathrm{N}, \mathrm{N}$-tris(benzimidazol-2yl-methyl) amine, J. Mol. Model. 21 (2015) 2759, doi:http://dx.doi.org/10.1007/s00894015-2759-1.

[27] K.N. Houk, L.L. Munchausen, Ionization potentials, electron affinities, and reactivities of cyanoalkenes and related electron-deficient alkenes. A frontier molecular orbital treatment of cyanoalkene reactivities in cycloaddition, electrophilic, nucleophilic, and radical reactions, J. Am. Chem. Soc. 98 (1976) 937-946, doi:http://dx.doi.org/10.1021/ja00420a012.

[28] M.T.N. Asit, K. Chandra, Use of Local Softness for the Interpretation of Reaction Mechanisms, Int. J. Mol. Sci. (2002), doi:http://dx.doi.org/10.3390/i3040310.

[29] E.G. Lewars, Computational Chemistry, Springer, Netherlands, Dordrecht, 2011. (accessed December 26, 2015) http://link.springer.com/10.1007/978-90-4813862-3.

[30] M.J. Frisch, G.W. Trucks, H.B. Schlegel, G.E. Scuseria, M.A. Robb, J.R. Cheeseman, et al., Gaussian, 09, Gaussian, Inc., Wallingford, CT, USA, 2009.

[31] L. Fotouhi, M. Zabeti, Electrochemical Oxidation of Sulfamethazine on MultiWalled Nanotube Film Coated Glassy Carbon Electrode, J. NanoStructures 4 (2014) 161-166.

[32] H. Li, J. Gupta, S. Wang, N. Zhang, C. Bubeck, Photoreduction of graphene oxide with polyoxometalate clusters and its enhanced saturable absorption, J. Colloid Interface Sci. 427 (2014) 25-28, doi:http://dx.doi.org/10.1016/j. jcis.2013.11.030.

[33] Y. Liu, J. Wang, Degradation of sulfamethazine by gamma irradiation in the presence of hydrogen peroxide, J. Hazard. Mater. 250-251 (2013) 99-105, doi: http://dx.doi.org/10.1016/j.jhazmat.2013.01.050.

[34] J. Gao, C. Hedman, C. Liu, T. Guo, J.A. Pedersen, Transformation of Sulfamethazine by Manganese Oxide in Aqueous Solution, Environ. Sci. Technol. 46 (2012) 2642-2651, doi:http://dx.doi.org/10.1021/es202492 h.

[35] S.O. Youhei Takeda, A Practical Synthesis of Azobenzenes Through Oxidative Dimerization of Aromatic Amines Using tert-Butyl Hypoiodite, Synthesis. 45 (2013) 1029-1033, doi:http://dx.doi.org/10.1055/s-0032-1318388.

[36] G.V. Boyd, Synthesis and Reactions of Hydrazo, Azo and Azoxy Compounds, in: S. Pataiessor (Ed.), Chem. Hydrazo Azo Azoxy Groups, John Wiley \& Sons, Ltd, 1997, 2016, pp. 301-366. (accessed December 26, 2015) http://onlinelibrary. wiley.com/doi/10.1002/0470023503.ch10/summary.

[37] M. Wang, K. Funabiki, M. Matsui, Synthesis and properties of bis(hetaryl) azo dyes, Dyes Pigments 57 (2003) 77-86, doi:http://dx.doi.org/10.1016/S01437208(03) 00011-1. 